\title{
Inteligencia emocional y rendimiento académico en estudiantes de Medicina Humana de la Universidad Nacional de la Amazonía Peruana
}

\author{
Emotional Intelligence and Academic Achievement in Human Medicine students of \\ Universidad Nacional de la Amazonía Peruana
}

\author{
Edwin Villacorta Vigoa*
}

http://dx.doi.org/10.21503/CienciayDesarrollo.2010.v12.04

\section{RESUMEN}

La presencia o ausencia de las capacidades y habilidades personales que contribuyen a la eficacia en el trato con los demás, denominada inteligencia emocional, puede hacer que las relaciones humanas tengan éxito o fracasen, influyendo directamente en el bienestar general y en la salud emocional.

Para estudiar la relación entre la inteligencia emocional y el rendimiento académico de los estudiantes de la Facultad de Medicina Humana de la Universidad Nacional de la Amazonía Peruana (FMH - UNAP), se realizó una investigación descriptiva-correlacional de tipo transeccional. Se administró el inventario de Bar - On, inventario de coeficiente emocional (I- CE) y se analizaron los récords académicos de todos los estudiantes del último nivel y ciclo de la FMH - UNAP, que totalizan 38; 22 del género masculino y 16 del género femenino. El 52,64\% está entre los 20 y 26 años de edad, y el 47,36\% tiene más de 27 años de edad.

Los resultados evidencian que en los estudiantes investigados predomina significativamente el nivel de inteligencia emocional promedio o adecuado (CEP); son $33(86,8 \%)$ los que se ubican en él. En todos los componentes de la inteligencia emocional, fueron $3(7,9 \%)$ y $2(5,3 \%)$ estudiantes los que se ubicaron en los niveles alto (CEA) y bajo (CEB) respectivamente, siendo 29 (76\%) los estudiantes que alcanzaron el nivel promedio en los componentes estado de ánimo en general y adaptabilidad; 26 (68\%) lograron dicho nivel en los componentes intrapersonal y manejo de estrés, mientras que 25 (65,79\%) lo hicieron en el componente relaciones interpersonales. El rendimiento académico promedio general es regular (11 a 14,99).

Se determinó que existe una correlación significativa entre la inteligencia emocional y el rendimiento académico de los estudiantes materia de investigación.

Palabras clave: inteligencia emocional, rendimiento académico.

\section{ABSTRACT}

The presence or absence of personal capabilities and skills contributing to get on with other people, the so called emotional intelligence can make human relations successful or unsuccessful, influencing directly to the general wellbeing and emotional health.

The purpose of the study was to determine the relationship between emotional intelligence and academic performance of students of the Faculty of Medicine of the National University of the Peruvian Amazon (FMH - UNAP). A descriptive-correlational research of transectional type was carried out. Bar-On inventory and emotional coeficient (EC-I) were applied to 38 promotional students and their

\footnotetext{
* Profesor de prostodoncia parcial removible y clínica estomatológica.
} 
academic records were analysed; 22 were male and 16 female students; $52,64 \%$ were between 20 and 26 years old and $47,36 \%$ were older than 27.

Results show a significant predominance of the average or adequate emotional intelligence level (AEQ) with $33(86,8 \%)$ in all components of the emotional intelligence. $3(7,9 \%)$ and $2(5,3 \%)$ students presented high emotional coefficient (HEC) and low emotional coefficient ( LEC), respectively. 29 (78\%) students reached the average level in the states of mind in general and of adaptability. 26 (68\%) students reached such a level in intrapersonal and stress management components while 25 (65,79\%) reached that level only in the interpersonal relationships component. The average academic perfomance is regular (11 a 14.99).

A significant correlation between emotional intelligence and academic performance wad found in the population studied.

Key words: emotional intelligence, academic efficiency.

\section{INTRODUCCIÓN}

Es ancestral la costumbre de relacionar la inteligencia con la capacidad de raciocinio lógico, con las competencias de comprensión, análisis, síntesis, resolución de problemas, etc., que componen el cociente intelectual. Sin embargo, desde finales del siglo pasado (1980 - Howard Gardner), otros conceptos se desarrollaron, apareciendo en 1990 (Peter Salovey y John Mayer) el concepto de inteligencia emocional. Posteriormente, en 1995, Daniel Goleman sustenta que es necesario estudiar la inteligencia humana resaltando la importancia del uso y gestión del mundo emocional y social para comprender el curso de la vida de las personas, suscitando así un gran interés en el ámbito educativo, debido a que ejerce influencia en el funcionamiento escolar de los alumnos.

Los docentes que orientan la formación de los futuros profesionales que egresan de las instituciones de educación superior se preocupan constantemente por conseguir optimizar los logros de sus estudiantes, realizando investigaciones que tienen como objetivo el análisis de las relaciones entre el rendimiento académico y diversas variables. En ellas se destacan las que analizan el papel de la inteligencia emocional en el rendimiento académico, las cuales evidencian que los alumnos emocionalmente inteligentes como norma general pueden llegar a obtener un mayor rendimiento escolar.

Los logros académicos que exhiben los estudiantes de la Facultad de Medicina Humana que cursan el internado (2007) no son los esperados, evidenciando aprendizajes regularmente logrados. Conocer el nivel de inteligencia emocional que muestran los estudiantes de la Facultad de Medicina Humana de la Universidad Nacional de la Amazonía Peruana y confrontarlo con su rendimiento académico permitirá obtener fundamentos para establecer la contribución de aquella en este, favoreciendo la búsqueda de soluciones para elevar los aprendizajes de los estudiantes.

La investigación, realizada en el año 2007, tuvo como propósito determinar la relación existente entre la inteligencia emocional y el rendimiento académico de los estudiantes de la Facultad de Medicina Humana de la Universidad Nacional de la Amazonía Peruana.

El trabajo está estructurado en siete capítulos. El primero presenta el planteamiento del problema e incluye objetivos de la investigación, justificación, importancia y limitaciones; el 
segundo, denominado marco teórico, contiene antecedentes del estudio, planteamientosteóricos o bases teóricas de las variables estudiadas, definición de términos básicos, hipótesis, modelo de investigación utilizado y variables del estudio; el tercero comprende metodología de la investigación, tipo de investigación, diseño metodológico (universo, técnicas e instrumentos y fuentes de recolección de datos utilizados, caracterización de la población y muestra), descripción de técnicas de procesamiento de datos y de análisis e interpretación de la información, contrastación de hipótesis, diseño estadístico y evaluación de hipótesis; el cuarto muestra los resultados obtenidos; en el quinto, se discuten y analizan los resultados; en el sexto, se presentan las conclusiones a las que arribamos en base a los resultados del estudio; en el séptimo, se alcanzan las recomendaciones que se formulan. Finalmente, se presenta la bibliografía revisada.

\section{MATERIALES Y MÉTODOS}

El estudio fue básico pero no experimental, descriptivo, de tipo transeccional-correlacional, cuya población objetivo estuvo constituida por la totalidad de alumnos de la Facultad de Medicina Humana de la UNAP que cursaron las asignaturas de Formación Profesional Especializada en el primer semestre académico 2007, que totalizan 256, siendo 108 del género femenino y 146 del masculino; la muestra seleccionada intencionalmente estuvo conformada por 38 estudiantes que cursaban el internado (2007), siendo 16 del género femenino y 22 del masculino, cuyas edades están entre 20 y 40 años.

La técnica para recolectar la información para la variable $\mathrm{X}$ : inteligencia emocional fue la observación indirecta, utilizando el inventario de Bar - On, inventario de cociente emocional (I - CE) que fue adoptado y estandarizado por Escurra y Cols, demostrando su confiabilidad en una muestra de Lima Metropolitana; para la variable rendimiento académico $(\mathrm{Y})$ se hizo uso de la técnica documental a través del inventario o récord académico de cada estudiante sujeto del estudio. Para las variables extrañas se apeló a la observación indirecta, utilizando el inventario.

El análisis estadístico hizo uso de técnicas de la estadística descriptiva, a través de frecuencias y proporciones.

\section{RESULTADOS}

\section{Inteligencia emocional}

Inteligencia emocional predominante de los estudiantes de la Facultad de Medicina Humana de la Universidad Nacional de la Amazonía Peruana.

Tabla 1. Niveles de inteligencia emocional global o total (CET) de los estudiantes de la Facultad de Medicina Humana de la UNAP

\begin{tabular}{|c|c|c|}
\hline Niveles de inteligencia emocional (CET) & Estudiantes & Porcentaje \\
\hline CEMB: Marcadamente alta (130 a más) & 00 & 0,0 \\
\hline CEA: Alto o muy alto (115 a 129) & 03 & 7,9 \\
\hline CEP: Promedio adecuado (85 a 114) & 33 & 86,8 \\
\hline CEB: Bajo (70 a 84) & 02 & 5,3 \\
\hline CEMB: Muy bajo (menos de 70) & 00 & 0,0 \\
\hline Total & 38 & 100,00 \\
\hline
\end{tabular}


En la tabla 1 se exhiben los resultados globales obtenidos mediante el inventario de Bar - On (I-CE) para los niveles de inteligencia emocional de los estudiantes de la Facultad de Medicina Humana de la Universidad Nacional de la Amazonía Peruana.

Como puede observarse, el cociente o nivel de inteligencia emocional total que predomina de manera acentuada en los estudiantes de Medicina Humana de la Universidad Nacional de la Amazonía Peruana es el promedio (86,8\%), y solo un $7,9 \%$ se ubica en el nivel alto, y un 5,3\% en el nivel bajo.

Para probarsi los niveles totales de inteligencia emocional son diferenciados, se utilizó la prueba no paramétrica de Friedman, cuyo resultado para un GL de 3 y una probabilidad de rechazo $p=0,000(p<0,05)$ fue $X 2 c=88,50$, lo que indica que estadísticamente existe una diferencia significativa entre los niveles totales de inteligencia emocional de los estudiantes de la Facultad de Medicina Humana de la Universidad Nacional de la Amazonía Peruana, resaltando el nivel promedio.

Inteligencia emocional de los estudiantes de la Facultad de Medicina Humana de la Universidad Nacional de la Amazonía Peruana por componentes de la inteligencia emocional

En relación al componente intrapersonal (tabla 2), se aprecia que globalmente un 68,42\% (26) logró puntajes que lo ubican en el nivel de capacidad emocional promedio (CEP). Solo un 18,42\% (7) está en el nivel de capacidad emocional alta (CEA), un 10,53\% (4) en el nivel bajo (CEB), y un 2,6\% (1) en muy bajo (CEMB).

Los puntajes más altos obtenidos por los sujetos del estudio en el nivel de capacidad emocional alta (CEA) se ubican en las habilidades de autoconcepto (26,3\%), autorrealización $(23,7 \%)$ e independencia $(23,7 \%)$, y el puntaje más bajo lo obtuvieron tres estudiantes $(7,9 \%)$ en el subcomponente comprensión emocional de sí mismo. Solo un estudiante (2,6\%) logró puntajes que lo ubican en la capacidad emocional marcadamente desarrollada (CEMD) en las habilidades de independencia.

$\mathrm{Al}$ analizar los niveles en los que se ubican los sujetos del estudio en cada uno de los cinco subcomponentes del intrapersonal, apreciamos que:

a. En la habilidad de ser asertivos (AS), capaces de percatarse y comprender sus sentimientos y emociones, diferenciándolos, conociendo el porqué de ellos, capaces de expresar sus sentimientos, creencias y pensamientos sin dañar los sentimientos de los demás, defender sus derechos de una manera no destructiva, un 24 (63,2\%) se ubica en el nivel de capacidad emocional promedio (CEP), 6 $(15,8 \%)$ se ubican en el nivel de bueno o alto,

Tabla 2. Componente intrapersonal (CIA) de la inteligencia emocional de los estudiantes de la Facultad de Medicina Humana de la Universidad Nacional de la Amazonía Peruana

\begin{tabular}{|c|c|c|c|c|c|c|c|c|c|c|c|c|}
\hline & \multicolumn{2}{|c|}{$\begin{array}{c}\text { Comprensión } \\
\text { emocional de sí mismo } \\
(\mathrm{CM})\end{array}$} & \multicolumn{2}{|c|}{$\begin{array}{l}\text { Asertividad } \\
\text { (AS) }\end{array}$} & \multicolumn{2}{|c|}{$\begin{array}{l}\text { Autoconcepto } \\
\text { (AC) }\end{array}$} & \multicolumn{2}{|c|}{$\begin{array}{c}\text { Autorrealización } \\
\text { (AR) }\end{array}$} & \multicolumn{2}{|c|}{$\begin{array}{l}\text { Independencia } \\
\text { (IN) }\end{array}$} & \multicolumn{2}{|c|}{$\%$ CIA } \\
\hline & Estudiantes & $\%$ & Estudiantes & $\%$ & Estudiantes & $\%$ & Estudiantes & $\%$ & Estudiantes & $\%$ & Estudiantes & $\%$ \\
\hline CEMB & 0 & 0 & 0 & 0 & 0 & 0 & 0 & 0 & 1 & 2,6 & 0 & 0 \\
\hline CEA & 3 & 7,9 & 6 & 15,8 & 10 & 26,3 & 9 & 23,7 & 9 & 23,7 & 7 & 18,42 \\
\hline CEP & 26 & 68,4 & 24 & 63,2 & 26 & 68,4 & 26 & 68,4 & 26 & 68,4 & 26 & 68,42 \\
\hline CEB & 6 & 15,8 & 8 & 21,0 & 2 & 5,3 & 3 & 7,9 & 2 & 5,2 & 4 & 10,53 \\
\hline CEMB & 3 & 7,9 & 0 & 0 & 0 & 0 & 0 & 0 & 0 & 0 & 1 & 2,6 \\
\hline TOTAL & 38 & 100 & 38 & 100 & 38 & 100 & 38 & 100 & 38 & 100 & 38 & 100 \\
\hline
\end{tabular}


y $8(21,0 \%)$ lo hacen en el nivel bajo o que requiere mejora.

b. En la capacidad emocional, que implica comprensión emocional de sí mismo o autoconcepto, comprendiendo, aceptando y respetándose a sí mismo, aceptando sus aspectos positivos y negativos, como sus limitaciones y posibilidades o en el área del autoconcepto (AC), son $26(68,4 \%)$ los que se ubican en el nivel de capacidad emocional promedio, $10(26,3 \%)$ se ubican en el nivel alto bueno y $2(5,3 \%)$ se ubicaron en el nivel bajo o por debajo del promedio.

c. En la habilidad de autorrealización (AR), que caracteriza a los individuos que saben lo que quieren o sus metas, adónde se dirigen y por qué, capaces de realizar lo que realmente pueden y quieren, disfrutando de hacerlo, son $26(68,4 \%)$ los que se ubicaron en el nivel de capacidad emocional, $9(23,7 \%)$ lo hacen en el nivel de bueno o alto y $3(7,9 \%)$ están en el nivel por debajo del promedio.

d. En el subcomponente de independencia (IN), que identifica a las personas que confían en sí mismas, autónomas e independientes en su forma de pensar y actuar, son $26(68,4 \%)$ las que se ubicaron en el nivel de capacidad promedio, 9 (23,7\%) lo están en el nivel bueno o alto o de capacidad emocional muy desarrollada, $1(2,6 \%)$ se ubicó en el nivel marcadamente alto (capacidad inusual), y 2
$(5,2 \%)$ se ubicaron en el nivel bajo.

e. En la habilidad de comprensión emocional de sí mismo (CM), que caracteriza a los individuos que se encuentran en contacto con sus sentimientos y emociones, saben lo que están sintiendo y entienden por qué sienten de esa manera, son 26 (98,4\%) los que se ubicaron en el nivel de inteligencia promedio, $3(7,9 \%)$ se ubicaron en el nivel bueno o alto, $6(15,8 \%)$ ocuparon el nivel bajo, y 1 (2,6\%) se ubicó en el nivel muy bajo.

Observamos que en el componente interpersonal (CEIE/CIE) (tabla y gráfico 03) en promedio, son 25 estudiantes $(65,79 \%)$ los que muestran un nivel de inteligencia emocional promedio (CEP), 8 (21\%) se ubican en el nivel bajo (CEB), 2 (5,3\%) en muy bajo y sólo $3(7,9 \%)$ lograron puntajes que los ubican en el nivel alto.

Apreciamos que de los tres subcomponentes que integran el componente interpersonal, es en el de relaciones interpersonales (RI) donde se obtuvieron los puntajes más altos $(27$, es decir, el 71,1\%), seguido del subcomponente responsabilidad social y empatía respectivamente (26 - 68,4\%; $23-60,5 \%)$, los tres ubicados en el nivel de capacidad emocional promedio.

Revisamos los niveles en los que se ubican los alumnos de la Facultad de Medicina Humana de

Tabla 3. Componente interpersonal (CEIE/CIE) de la inteligencia emocional de los estudiantes de la Facultad de Medicina Humana de la Universidad Nacional de la Amazonía Peruana

\begin{tabular}{|c|c|c|c|c|c|c|c|c|}
\hline & \multicolumn{2}{|c|}{$\begin{array}{c}\text { Empatía } \\
\text { (EM) }\end{array}$} & \multicolumn{2}{|c|}{$\begin{array}{c}\text { Relaciones } \\
\text { interpersonales } \\
\text { (RI) }\end{array}$} & \multicolumn{2}{|c|}{$\begin{array}{l}\text { Responsabilidad } \\
\text { social } \\
\text { (RS) }\end{array}$} & \multicolumn{2}{|c|}{$\% \mathrm{CIE}$} \\
\hline & Estudiantes & $\%$ & Estudiantes & $\%$ & Estudiantes & $\%$ & Estudiantes & $\%$ \\
\hline CEMB & 0 & 0 & 0 & 0 & 0 & 0 & 0 & 0 \\
\hline CEA & 4 & 10,5 & 3 & 7,9 & 1 & 2,6 & 3 & 7,9 \\
\hline CEP & 23 & 60,5 & 27 & 71,1 & 26 & 68,4 & 25 & 65,79 \\
\hline CEB & 10 & 26,3 & 5 & 13,2 & 8 & 21,1 & 8 & 21,0 \\
\hline CEMB & 1 & 2,6 & 3 & 7,9 & 3 & 7,9 & 2 & 5,3 \\
\hline TOTAL & 38 & 100 & 38 & 100 & 38 & 100 & 38 & 100 \\
\hline
\end{tabular}


la UNAP, en cada uno de los tres subcomponentes del interpersonal. Los resultados de la muestra exhiben que:

a. En la habilidad de empatía (EM), que distingue a los que son conscientes de los sentimientos de otros y los valoran, son sensibles a lo que los demás sienten y pueden comprenderlos, son $4(10,5 \%)$ los que alcanzaron puntuaciones que los ubican en el nivel de capacidad emocional alta, $23(60,5 \%)$ se ubicaron en la capacidad promedio, 10 (26,3\%) en el nivel bajo y $1(2,6 \%)$ en el nivel muy bajo.

b. En cuanto a la capacidad emocional de relaciones interpersonales (RI) que caracteriza a los individuos que son capaces de establecery mantener relaciones mutuamente satisfactorias, capaces de intimar con otros, además de dar y recibir afecto, son $3(7,9 \%)$ los que alcanzaron puntuaciones que los ubican en el nivel de capacidad emocional alta, 27 (71,1\%) los que se ubicaron en el nivel de capacidad emocional promedio, 5 $(13,2 \%)$ están en el nivel bajo y 3 (7,9\%) se ubicaron en el nivel muy bajo.

c. En la capacidad de responsabilidad social, (RS) que identifica a las personas confiables y responsables en un grupo social, que colaboran y cooperan, aun cuando no obtengan beneficios personales, se encontró que 1 estudiante (2,6\%) se ubica en el nivel de inteligencia emocional alto, 26 (68,4\%) alcanzaron puntuaciones que los ubican en el nivel promedio, $8(21,1 \%)$ están en el nivel bajo y $3(7,9 \%)$ en el muy bajo.

Los resultados obtenidos exhiben que:

a. De los estudiantes sujeto de la investigación que se ubicaron en el nivel de CIE baja, tienen niveles por mejorar en cuanto a empatía 10 $(26,3 \%)$, relaciones interpersonales $5(13,2 \%)$ y en responsabilidad social $8(21,1 \%)$.

b. Son tres $(7,9 \%)$ los estudiantes cuyas relaciones interpersonales y responsabilidad social respectivamente se ubican en el nivel de coeficiente emocional muy bajo y 1 (2,6\%) cuya empatía se ubica en igual nivel, por lo que necesitan mejorar dichos subcomponentes.

En la tabla 4 apreciamos que en el componente del manejo de estrés (CME), en promedio, el $68,42 \%$ de estudiantes alcanzó puntajes que los ubican en el nivel de inteligencia emocional adecuado promedio, nivel que se mantiene en los dos subcomponentes del manejo del estrés. Seis estudiantes se ubican en el nivel alto, 2 en el marcadamente alto, 3 en el bajo y 1 en el muy bajo.

Los resultados de la muestra permiten

Tabla 4. Componente del manejo del estrés (CEME/CME) de la inteligencia emocional de los estudiantes de la Facultad de Medicina Humana de la Universidad Nacional de la Amazonía Peruana

\begin{tabular}{l|c|c|c|c|c|c|} 
& \multicolumn{2}{c|}{$\begin{array}{c}\text { Tolerancia al estrés } \\
\text { (TE) }\end{array}$} & \multicolumn{2}{c|}{$\begin{array}{c}\text { Control de } \\
\text { impulsos (CI) }\end{array}$} & \multicolumn{2}{c}{ X CME } \\
\cline { 2 - 7 } & Estudiantes & $\%$ & Estudiantes & $\%$ & Estudiantes & $\%$ \\
\hline CEMB & 4 & 10,5 & 0 & 0 & 2 & 5,3 \\
\hline CEA & 4 & 10,5 & 7 & 18,4 & 6 & 15,78 \\
\hline CEP & 27 & 71,1 & 26 & 68,4 & 26 & 68,42 \\
\hline CEB & 3 & 7,9 & 4 & 10,5 & 3 & 7,9 \\
CEMB & 0 & 0 & 1 & 2,6 & 1 & 2,6 \\
\hline TOTAL & 38 & 100 & 38 & 100 & 38 & 100 \\
\hline
\end{tabular}


apreciar que los alumnos investigados exhiben cuatro niveles de inteligencia emocional en el componente del manejo del estrés:

a. En cuanto a la habilidad para soportar eventos adversos, situaciones estresantes y fuertes emociones sin "desmoronarse", enfrentando activa y positivamente el estrés (tolerancia al estrés), son 4 (10,5\%) los que se ubican en el nivel marcadamente alto y en el alto, 27 (71,1\%) están ubicados en el promedio o adecuado, y 03 (7,9\%), en el bajo.

b. Con respecto a la habilidad para resistir o postergar un impulso o tentaciones para actuar y controlar sus emociones (control de impulsos), son $7(18,4 \%)$ los que alcanzaron el nivel alto, $26(68,4 \%)$ se ubicaron en el nivel promedio o adecuado, 4 (10,5\%) están en el bajo, y 01 (2,6\%) en el muy bajo.

La tabla 5 presenta los resultados referidos al componente estado de ánimo en general (CEAG) de la inteligencia emocional de los estudiantes materia de investigación, apreciando que el 76,0\% (29) se ubica en el nivel promedio o adecuado, 18,42\% (7) (CEP) lo está en el nivel alto y 5,3\% (2) en el nivel bajo (CEB).

Al examinar los datos encontrados, apreciamos que los sujetos del estudio exhiben tres niveles de inteligencia emocional en los dos subcomponentes del estado de ánimo en general, ya que:

a. En la habilidad para sentirse satisfechos en la vida, para disfrutar de sí mismos y de otros, para divertirse y expresar sentimientos positivos (felicidad), son $6(15,8 \%)$ los que se ubican en el nivel de coeficiente emocional alto, $28(73,7 \%)$ en el nivel promedio o adecuado y $04(10,5 \%)$ en el nivel bajo.

b. En la habilidad para ver el aspecto más brillante de la vida y mantener una actitud positiva a pesar de la adversidad y los sentimientos negativos (optimismo), 1 $(2,6 \%)$ se ubicó en el nivel bajo; $30(78,9 \%)$ en el nivel promedio o adecuado y $07(18,4 \%)$ en el nivel alto.

La tabla 6 muestra los resultados obtenidos por los sujetos estudiados en el componente adaptabilidad. En ella apreciamos que 29 $(76,34 \%)$ se ubican en el nivel promedio o adecuado, 4 (10,53\%) se ubican en el nivel bajo (CEB), igual cantidad se ubica en el nivel de capacidad emocional alta y $1(2,6 \%)$ en el de muy bajo (CEMB).

Analizando los resultados de la muestra, en cada subcomponente de adaptabilidad

Tabla 5. Componente del estado de ánimo en general (CEAG/CAG) de la inteligencia emocional de los estudiantes de la Facultad de Medicina Humana de la Universidad Nacional de la Amazonía Peruana

\begin{tabular}{l|c|c|c|c|c|c} 
& \multicolumn{2}{c|}{$\begin{array}{c}\text { Felicidad } \\
(\mathrm{FE})\end{array}$} & \multicolumn{2}{c|}{$\begin{array}{c}\text { Optimismo } \\
(\mathrm{OP})\end{array}$} & \multicolumn{2}{c}{ X CAG } \\
\cline { 2 - 7 } & Estudiantes & $\%$ & Estudiantes & $\%$ & Estudiantes & $\%$ \\
\hline CEMD & 0 & 0 & 0 & 0 & 0 & 0 \\
\hline CEA & 6 & 15,8 & 7 & 18,4 & 7 & 18,4 \\
\hline CEP & 28 & 73,7 & 30 & 78,9 & 29 & 76,6 \\
\hline CEB & 4 & 10,5 & 1 & 2,6 & 2 & 5,3 \\
CEMB & 0 & 0 & 0 & 0 & 0 & 0 \\
\hline TOTAL & 38 & 100 & 38 & 100 & 38 & 100 \\
\hline
\end{tabular}


apreciamos que los estudiantes de Medicina Humana de la Universidad Nacional de la Amazonía Peruana (UNAP) exhiben lo siguiente:

a. En la habilidad para reconocer y definir problemas, así como para generar e implementar soluciones potencialmente efectivas (solución de problemas), son 4 $(10,5 \%)$ los que se ubican en el nivel alto, $31(81,6 \%)$ en el promedio o adecuado, y 3 $(7,9 \%)$ en el nivel bajo. b. Cuatro subniveles en el subcomponente que destaca a los individuos que son capaces de evaluar la correspondencia entre lo que experimentan o lo subjetivo y lo que en realidad existe o lo objetivo (prueba de la realidad). Son 4 (10,5\%) los que se ubican en el nivel alto, 29 (76,3\%) están en el nivel promedio o adecuado, 2 (5,3\%) en el bajo y 3 $(7,9 \%)$ en el muy bajo.

c. En la habilidad y capacidad para adecuar sus emociones, sentimientos, pensamientos y comportamientos a situaciones y condiciones

Tabla 06. Componente adaptabilidad (CAD/CEAD) de la inteligencia emocional de los estudiantes de la Facultad de Medicina Humana de la Universidad Nacional de la Amazonía Peruana

\begin{tabular}{l|c|c|c|c|c|c|c|c|} 
& \multicolumn{2}{c|}{$\begin{array}{c}\text { Solución de } \\
\text { problemas (SP) }\end{array}$} & $\begin{array}{c}\text { Prueba de la realidad } \\
\text { (PR) }\end{array}$ & \multicolumn{2}{c|}{ Flexibilidad (FL) } & \multicolumn{2}{c}{$\begin{array}{c}\text { X } \\
\text { CEAD/CAD }\end{array}$} \\
\cline { 2 - 11 } & Estudiantes & $\%$ & Estudiantes & $\%$ & Estudiantes & $\%$ & Estudiantes & $\%$ \\
\hline CEMB & 0 & 0 & 0 & 0 & 0 & 0 & 0 & 0 \\
\hline CEA & 4 & 10,5 & 4 & 10,5 & 4 & 10,5 & 4 & 10,53 \\
\hline CEP & 31 & 81,6 & 29 & 76,3 & 28 & 73,7 & 29 & 76,34 \\
CEB & 3 & 7,9 & 2 & 5,3 & 5 & 13,2 & 4 & 10,53 \\
\hline CEMB & 0 & 0 & 3 & 7,9 & 1 & 2,6 & 1 & 2,6 \\
\hline TOTAL & 38 & 100 & 38 & 100 & 38 & 100 & 38 & 100 \\
\hline
\end{tabular}

Tabla 7. Niveles de inteligencia emocional y edad de los estudiantes de la Facultad de Medicina Humana de la Universidad Nacional de la Amazonía Peruana

\begin{tabular}{|c|c|c|c|c|c|c|c|c|c|c|c|c|c|c|}
\hline \multirow{2}{*}{$\begin{array}{l}\text { Niveles de inteligencia } \\
\text { emocional (CET) }\end{array}$} & \multicolumn{12}{|c|}{ EDADES } & \multirow{2}{*}{ TOTAL } & \multirow{2}{*}{$\%$} \\
\hline & 23 & 24 & 25 & 26 & 27 & 28 & 29 & 30 & 31 & 33 & 36 & 40 & & \\
\hline $\begin{array}{l}\text { CEMD: } \\
\text { Marcadamente alta } \\
\text { (130 a más) }\end{array}$ & 0 & 0 & 0 & 0 & 0 & 0 & 0 & 0 & 0 & 0 & 0 & 0 & 0 & 0 \\
\hline $\begin{array}{l}\text { CEA: alta o muy alta } \\
(115 \text { a } 129)\end{array}$ & 0 & 0 & 1 & 0 & 0 & 0 & 1 & 0 & 0 & 0 & 0 & 1 & 3 & 7,89 \\
\hline $\begin{array}{l}\text { CEP: Promedio, } \\
\text { adecuado ( } 85 \text { a 114) }\end{array}$ & 2 & 4 & 8 & 3 & 5 & 4 & 0 & 2 & 2 & 1 & 2 & 0 & 33 & 86,85 \\
\hline CEB: Bajo (70 a 84) & 1 & 0 & 1 & 0 & 0 & 0 & 0 & 0 & 0 & 0 & 0 & 0 & 2 & 5,26 \\
\hline $\begin{array}{l}\text { CEMB: Muy bajo } \\
\text { (Menos de } 70)\end{array}$ & 0 & 0 & 0 & 0 & 0 & 0 & 0 & 0 & 0 & 0 & 0 & 0 & 0 & 0 \\
\hline TOTAL & 3 & 4 & 10 & 3 & 5 & 4 & 1 & 2 & 2 & 1 & 2 & 1 & 38 & 100 \\
\hline
\end{tabular}


cambiantes, un alto porcentaje $(73,7 \%$, es decir, 28 estudiantes) se ubica en el nivel promedio o adecuado. Cuatro estudiantes (10,5\%) están en el nivel alto, 5 (13,2\%) están en el nivel bajo, y uno (2,6\%), en el nivel muy bajo.

Inteligencia emocional en función de características personales

\section{Edad}

La tabla 7 exhibe los niveles de inteligencia emocional en los sujetos estudiados, diferenciados según edad, datos obtenidos mediante el inventario de Bar On (I - CE); en ella observamos:

a. 38 participantes están entre 23 y 40 años de edad, predominando los que tienen 25 años (10), siguen los que tienen 27 años (05), luego están los de 28 y 24 años respectivamente (04 cada uno); después están los que tienen 26 y 23 años (3 cada uno), prosiguen los de 30, 31 y 36 años ( 2 cada uno), y finalmente los que tienen 29, 33 y 40 años ( 1 cada uno).

b. Que los participantes, teniendo diferentes edades, en un $86,85 \%$ (33) se ubican en el nivel de inteligencia emocional promedio o adecuado; solo un $7,89 \%$ (03) se ubica en el nivel alto y el 5,26\% (02) lo hace en el nivel bajo. Debemos precisar que la edad no influye significativamente en el nivel de inteligencia emocional.

Atendiendo a los años o edad que tienen los sujetos de estudio, hemos establecido tres grupos etáreos: hasta 26 años, de 27 a 30 años y más de 30 años.

La tabla 8 permite apreciar que son los estudiantes del grupo etáreo entre 27 a 30 años los que alcanzaron un mayor porcentaje $(91,6 \%)$ en el nivel promedio de inteligencia emocional; se ubican luego los que tienen hasta 26 años $(85 \%)$ y finalmente los que tienen más de 30 años $(83,33 \%)$. Observamos también que en cada uno de los tres grupos hay un estudiante que alcanzó el nivel alto de inteligencia emocional.

Tabla 8. Niveles de inteligencia emocional de los estudiantes de la Facultad de Medicina Humana de la Universidad Nacional de la Amazonía Peruana, diferenciados por grupos etáreos

\begin{tabular}{|l|l|c|c|}
\multirow{2}{*}{ GRUPOS ETÁREOS } & \multicolumn{3}{|c|}{ NIVELES DE INTELIGENCIA EMOCIONAL } \\
\cline { 2 - 4 } & & FRECUENCIA & $\%$ \\
\hline \multirow{3}{*}{ Hasta 26 años } & CEA & 1 & 5 \\
& CEP & 17 & 85 \\
& CEB & 2 & 10 \\
& TOTAL & 20 & 100 \\
\hline \multirow{3}{*}{ De 27 a 30 años } & CEA & 1 & 8,4 \\
& CEP & 11 & 91,6 \\
& CEB & 0 & 0 \\
& TOTAL & 12 & 100 \\
\hline \multirow{3}{*}{ Más de 30 años } & CEA & 1 & 16,67 \\
& CEP & 5 & 83,33 \\
& CEB & 0 & 0 \\
\hline
\end{tabular}




\section{Sexo / Género}

En la tabla 9 se aprecia a los estudiantes investigados según género y diferenciados por el nivel de inteligencia alcanzado. Observamos que son $22(57,89 \%$ del total $)$ estudiantes del sexo masculino y $16(42,11 \%$ del total $)$ del sexo femenino.

Apreciamos también que del 100\% (38) de los sujetos de la muestra, los estudiantes del sexo masculino son los que alcanzan mayores porcentajes en el nivel de inteligencia promedio (43,37\%: 18 de 22), mientras que las alumnas del sexo femenino lograron el 39,48\% (15 a 16). Se evidencia también que son los estudiantes del sexo masculino los que lograron niveles de inteligencia alta: 3 (7,89\% del 100\%).

Al realizar el análisis de la tabla 10 , se aprecia que al diferenciar los datos por género se evidencia que son las alumnas del sexo femenino las que obtuvieron mayores porcentajes en el nivel de inteligencia promedio (93,75\%), mientras que los estudiantes del sexo masculino obtuvieron mayores porcentajes en el nivel alto (13,64\%).

Rendimiento académico de los estudiantes de la Facultad de Medicina Humana de la

\section{Universidad Nacional de la Amazonía Peruana}

En la tabla 11 se muestra el rendimiento académico por categorías de los estudiantes de la Facultad de Medicina Humana de la Universidad Nacional de la Amazonía Peruana (UNAP), traducido en promedio ponderado anual (PPA).

Se aprecia que los estudiantes de Medicina Humana de la UNAP tienen rendimiento académico anual diferenciado en dos categorías: bueno y regular. Son 35 (92,2\%) los alumnos que se ubican en la categoría de regular o bajo y $3(7,8 \%)$ los que se ubican en la categoría bueno. La media anual es de 13,46.

\section{Relación entre la inteligencia emocional y} el rendimiento académico de los estudiantes de la Facultad de Medicina Humana de la Universidad Nacional de la Amazonía Peruana

Se observa que de los 3 estudiantes con PPA bueno, $02(66,67 \%)$ se ubican en el nivel de inteligencia emocional promedio, y 1 (33,33\%) en el alto. Observamos diferente distribución en el rendimiento académico regular, de modo que el $88,58 \%$ (31) obtuvo puntajes que lo ubican en el nivel de inteligencia promedio, y 5,71\% (02) se ubica en el nivel de inteligencia alto.

Tabla 9. Niveles de inteligencia emocional y género de los estudiantes de la Facultad de Medicina Humana de la Universidad Nacional de la Amazonía Peruana

\begin{tabular}{l|c|c|c|c|}
\multirow{2}{*}{ Niveles de inteligencia emocional (CET) } & \multicolumn{2}{|c|}{ Género } & \multicolumn{2}{c}{ Porcentaje } \\
\cline { 2 - 5 } & Masculino & Femenino & Masculino & Femenino \\
\hline CEMD: Marcadamente alta (130 a más) & 0 & 0 & 0 & 0 \\
\hline CEA: Alta o muy alta (115 a 129) & 3 & 0 & 7,89 & 0 \\
\hline CEP: Promedio, adecuado (85 a 114) & 18 & 15 & 47,37 & 39,48 \\
\hline CEB: Bajo (70 a 84) & 1 & 1 & 2,63 & 2,63 \\
\hline CEMB: Muy bajo (Menos de 70) & 0 & 0 & 0 & 0 \\
\hline Total parcial & 22 & 16 & 57,89 & 42,11 \\
\hline Total general & & 38 & & 100 \\
\hline
\end{tabular}


Tabla 10. Niveles de inteligencia emocional de los estudiantes de la Facultad de Medicina Humana de la Universidad Nacional de la Amazonía Peruana, diferenciados por género

\begin{tabular}{l|l|c|c|}
\multirow{2}{*}{ Género } & \multicolumn{3}{|c}{ Niveles de inteligencia emocional } \\
\cline { 2 - 4 } & & Frecuencia & $\%$ \\
\hline \multirow{3}{*}{ Masculino } & CEA & 3 & 13,64 \\
& CEP & 18 & 81,82 \\
& CEB & 1 & 4,54 \\
& TOTAL & 22 & 100 \\
\hline \multirow{3}{*}{ Femenino } & CEA & 0 & 0 \\
& CEP & 15 & 93,75 \\
& CEB & 1 & 6,25 \\
\hline
\end{tabular}

Tabla 11. Rendimiento académico anual categorizado de los estudiantes de la Facultad de Medicina Humana de la Universidad Nacional de la Amazonía Peruana

\begin{tabular}{l|c|c|c|}
\multirow{2}{*}{ Rendimiento académico categorizado } & \multicolumn{2}{|c|}{ Promedio ponderado anual } & \multirow{2}{*}{ Media anual } \\
\cline { 2 - 3 } & $N^{\circ}$ & $\%$ & \\
\hline Eficiente (18 a 20) & 0 & 0 & \\
\hline Bueno (15 a 17,99) & 3 & 7,8 & 13,46 \\
\hline Regular, bajo (11 a 14,99) & 35 & 92,2 & \\
Deficiente (menos de 11) & 0 & 0 & \\
\hline Total & 38 & 100 & \\
\hline
\end{tabular}

Tabla 12. Inteligencia emocional y rendimiento académico según PPA de los estudiantes de la Facultad de Medicina Humana de la Universidad Nacional de la Amazonía Peruana

\begin{tabular}{|c|c|c|c|c|c|c|c|c|c|c|}
\hline \multirow{3}{*}{$\begin{array}{l}\text { NIVELES DE } \\
\text { INTELIGENCIA }\end{array}$} & \multicolumn{10}{|c|}{ RENDIMIENTO ACADÉMICO } \\
\hline & \multicolumn{2}{|c|}{ EFICIENTE } & \multicolumn{2}{|c|}{ BUENO } & \multicolumn{2}{|c|}{ REGULAR } & \multicolumn{2}{|c|}{ DEFICIENTE } & \multicolumn{2}{|c|}{ TOTAL } \\
\hline & $\mathrm{N}$ & $\%$ & $\mathrm{~N}$ & $\%$ & $\bar{N}$ & $\%$ & $\mathrm{~N}$ & $\%$ & $\mathrm{~N}$ & $\%$ \\
\hline CEMD & 0 & 0 & 0 & 0 & 0 & 0 & 0 & 0 & 0 & 0 \\
\hline CEA & 0 & 0 & 1 & 33,33 & 2 & 5,71 & 0 & 0 & 3 & 7,89 \\
\hline CEP & 0 & 0 & 2 & 66,67 & 31 & 88,58 & 0 & 0 & 33 & 86,85 \\
\hline CEB & 0 & 0 & 0 & 0 & 2 & 5,71 & 0 & 0 & 2 & 5,26 \\
\hline CEMB & 0 & 0 & 0 & 0 & 0 & 0 & 0 & 0 & 0 & 0 \\
\hline TOTAL & 0 & 0 & 3 & 100 & 35 & 100 & 0 & 0 & 38 & 100 \\
\hline
\end{tabular}

$$
\mathrm{X} 2 \mathrm{C}=18,77 \quad \mathrm{gl}=1 \quad \mathrm{p}=0,001(\mathrm{p}>0,05)
$$


Para verificar si existe relación entre la inteligencia emocional y el rendimiento académico según el promedio ponderado anual acumulado de los estudiantes de Medicina Humana de la Universidad Nacional de la Amazonía Peruana (UNAP), se aplicó la prueba no paramétrica Chi cuadrada, cuyo resultado para $\mathrm{gl}=1$ y una probabilidad de rechazo $\mathrm{p}>$ 0,05 fue de $\mathrm{X} 2 \mathrm{c}=18,77$ indica que existe relación estadísticamente significativa entre las variables inteligencia emocional y rendimiento académico en PPA de los estudiantes de la Facultad de Medicina Humana de la Universidad Nacional de la Amazonía Peruana (UNAP).

\section{DISCUSIÓN}

1. Es innegable que los individuos se adaptan o afrontan las demandas y presiones del ambiente de forma distinta. A cualquier edad y en cualquier cultura tienen y utilizan diversas competencias, habilidades y destrezas para manejar el estrés, establecer relaciones interpersonales, dirigir sus emociones y estados de ánimo, adaptándose y ajustándose al medio a fin de conseguir resultados académicos óptimos.

En la tarea de optimizar la calidad del proceso educativo, reviste particular importancia reflexionar sobre una de las variables que influyen en el rendimiento académico de los estudiantes: su inteligencia emocional. Aquí mostramos la relación entre la inteligencia emocional y el rendimiento académico.

2. En los resultados observamos en primer lugar que el nivel de inteligencia emocional global o total (CET) de los estudiantes investigados se encuentra ubicado mayoritariamente en el nivel promedio $(66,8 \%)$, lo que significa una capacidad emocional adecuada, típicamente saludable, la misma que les permitirá manejar las exigencias del entorno (Bar On, 1997) controlando sus emociones, comunicándose de forma activa y proyectando una actitud optimista ante la vida.

El $7,9 \%$ se encuentra en el nivel alto, lo que revela una capacidad emocional muy desarrollada, ya que se encuentra en contacto con sus sentimientos, se siente bien consigo mismo y generalmente tiene éxito para relacionarse con las demás personas. De otro lado, un 5,3\% (02) de los estudiantes se ubica en el nivel bajo, requiriendo apoyo para mejorar algunas competencias y habilidades particulares, a fin de que pueda enfrentar las exigencias del futuro.

Estos resultados son corroborados de alguna manera por Matalinares et al (2005), que al estudiar la inteligencia emocional en estudiantes de Lima Metropolitana encontraron que la media de los sujetos de la muestra se ubicó en el nivel promedio.

León (2001) contribuye cuando reporta que en el estudio de la inteligencia emocional en alumnos universitarios, usando la prueba de Bar On, el 100\% de los evaluados se ubica en el nivel promedio.

3. Al realizar el análisis de la inteligencia emocional por componentes de los estudiantes de la muestra (tablas 2, 3, 4, 5 y 6), encontramos que, en promedio, las competencias referidas al manejo de estado de ánimo en general (CEAG / CAG) ocupan los niveles más altos, seguidas muy de cerca por las de adaptabilidad al medio y manejo de estrés, estando luego las capacidades para dirigir sus emociones y establecer relaciones interpersonales.

Se evidencia una marcada tendencia de los sujetos del estudio para disfrutar de la vida, tener una perspectiva positiva de ella, sentirse contentos en general, tener un buen manejo 
delas dificultades diarias-del entorno, resistir al estrés sin "desmoronarse" o sin perder el control-, trabajar bajo presión-, sentirse bien consigo mismo y con lo que hacen en la vida, comprendiendo, interactuando y relacionándose muy bien con los demás. Son características que los muestran como personas optimistas, satisfechas con su propia vida, realistas y hábiles para reconocer, definir e intentar resolver problemas, capaces de adecuar sus emociones, sentimientos, pensamientos y comportamientos a situaciones y condiciones cambiantes; con habilidades para ser tolerantes al estrés y capaces de interaccionar positivamente con los demás.

4. El nivel de inteligencia emocional de los estudiantes de Medicina Humana de la UNAP, analizado en función de características personales (tablas 8, 9, 10 y 11), no evidencia diferencias significativas en función a edad, grupo etáreo y género de los sujetos del estudio.

Estos resultados son corroborados de alguna manera con los encontrados por Murata (2004), quien observó que no existen diferencias por sexo en la inteligencia emocional. Estos resultados son discordantes con los alcanzados por Velásquez (2003), que encontró diferencias en función del sexo, las cuales favorecen al sexo femenino en el componente referido a la habilidad para las relaciones interpersonales y control de impulsos, emociones o manejo del estrés.

5. En relación al rendimiento académico de los estudiantes, observamos que el 92,2\% (PPA) de los alumnos de la Facultad de Medicina Humana de la UNAP (tabla 12) se sitúa en la categoría de calificación regular, mientras que un $7,8 \%$ (PPA) se ubica en la categoría bueno.

\section{CONCLUSIONES}

\section{Conclusiones parciales}

1. El nivel o cociente emocional (CE) o nivel de inteligencia emocional (I-CE) total o global (CET) que predomina en los estudiantes de la Facultad de Medicina Humana de la UNAP es el promedio o adecuado (CEP), seguido por el alto o muy alto (CEA) y finalmente por el bajo (CEB).

2. Los sujetos del presente estudio, exhibiendo puntajes que los ubican en la capacidad emocional adecuada o promedio (CEP) en cada uno de los componentes o dimensiones del cociente emocional (CE), evidencian mayores fortalezas en la dimensión estado de ánimo general (CEAG), seguida por la de adaptabilidad (CAD), continuando la intrapersonal (CIA) y manejo de estrés (CME), ubicándose al final la interpersonal (CIE).

3. Los estudiantes de la Facultad de Medicina Humana de la UNAP, en términos generales, muestran potencialidades y fortalezas en las habilidades de los subcomponentes de optimismo (OP), solución de problemas (SP), autoconcepto (AC), tolerancia al estrés (TE) y relaciones interpersonales (RI), evidenciando debilidad en habilidades específicas de felicidad (FE), flexibilidad (FL), control de impulsos (CI), asertividad (AS) y empatía (EM).

4. Las características personales de edad, grupo etáreo y género no ejercen influencia significativa en el nivel de inteligencia emocional de los sujetos del estudio.

5. El rendimiento académico de los estudiantes de Medicina Humana de la Universidad Nacional de la Amazonía Peruana se sitúa mayoritariamente en la categoría de regular o bajo (PPA: 92,2\%), seguido de la de los ubicados en el nivel bueno (PPA: 7,8\%). Ningún estudiante se ubica en el nivel deficiente ni eficiente. 
6. Si se potencian o minimizan las fortalezas o debilidades detectadas en cada subcomponente del cociente emocional de los sujetos del estudio, en aras de contribuir al desarrollo de sus competencias y habilidades emocionales, se incrementará su funcionamiento general, enfrentando con éxito las exigencias del entorno.

\section{Conclusión general}

Existe relación estadísticamente significativa entre la inteligencia emocional y el rendimiento académico en los estudiantes de la Facultad de Medicina Humana de la Universidad Nacional de la Amazonía Peruana.

\section{RECOMENDACIONES}

\section{Recomendaciones parciales}

1. Las autoridades universitarias deben planificar, organizar, desarrollar y evaluar estrategias de capacitación sobre conceptos e identificación de inteligencia emocional destinadas a los docentes universitarios, a fin de apoyarlos en la identificación de las habilidades emocionales de relativa fortaleza o debilidad en su desempeño actual para mejorarlas y enfrentar con éxito las exigencias del entorno.

2. Las autoridades universitarias deben establecer, organizar y ejecutar estudios que permitan conocer las habilidades y destrezas personales, emocionales y sociales que influyen en la capacidad de los estudiantes para adaptarse y enfrentar las demandas y presiones del medio (IE). Conociéndolas, deberán establecer y desarrollar programas y estrategias que permitan potenciar o mejorar las habilidades y destrezas de su desempeño actual como estudiantes universitarios y en la profesión para la cual se forman en los claustros universitarios.

3. Es necesario que las autoridades universitarias promuevan la realización de estudios de seguimiento sobre las acciones que se realicen en todas las facultades o unidades académicas de la institución, conducentes al fortalecimiento de las diferentes habilidades emocionales de los estudiantes universitarios (IE).

\section{Recomendación general}

Estimular la realización de trabajos de investigación similares al presente en todo nivel educativo, a fin de obtener datos útiles sobre los niveles de inteligencia emocional de los educandos e integrarlos al desarrollo de las habilidades cognitivas y prácticas, en la búsqueda de soluciones para elevar la calidad educativa en general y el bienestar personal y social de la comunidad.

\section{REFERENCIAS BIBLIOGRÁFICAS}

1. Aceros, J. C. S. Angarita y O. Campos. (2003) Correlación entre depresión y rendimiento académico en los estudiantes de quinto grado de primaria del colegio la Santísima Trinidad. Editorial Bucaramanga, Colombia. 45 p.

2. Arbola G. y Pérez H. (2002) Inteligencia emocional: teoría y praxis en educación. Tesis doctoral. Universidad Centro Occidental Lisandro Alvarado, Barquisimeto.

3. Bar - On R. (1997) The emocional Quotient Inventory (EQI): Technical Manaul. Toronto. Multi - Health Systems.

4. Camarena R.M. (1985). "Reflexiones en torno al rendimiento escolar y la eficiencia terminal". Revista de Educación Superior, vol. 13 , No $01.53,34$ y ss.

5. Cardama R.V. El rendimiento escolar (serial on line). Citado el 20 de agosto de 2007. disponible en www.e.cristians.net.

6. Drago, J. M. (2005). The relationship between emocional intelligence and academia achievement in notraditional collage student. Disertation Abstracts International: Section B. The Sciences and Engineering. Vol 65 (9- 
B). 2005, 481 .

7. Escalante R. M. (2002). Programa de intervención de estrategias de aprendizaje en el marco de la metacognición, aplicado en la asignatura de lenguaje. I año, 2002. UNIFE. Lima,Perú. Tesis de ,aestría. 137 pp.

8. Escurra, M. y Cols. (2000). "Construcción de una prueba de investigación emocional". Revista de investigación en psicología. Vol. 3. No $01 . p p 71-85$.

9. Extremera PN., Fernández; Berrocal P. (2004). "El papel de la inteligencia emocional en el alumnado: evidencias empíricas". Revista Electrónica de Investigación Educativa, 6 (2). Consultado el 10 de octubre 2007 en http: //redie.uabc.mx/ vol. 6 No2

10. Fernández; Berrocal P., Extremara N., Ramos N. (2003. a) Inteligencia emocional y depresión. Encuestas en psicología social. 1 (5) $251-254$.

11. Gardner H. (1983) Frames of mind. New York, Básic Books.

12. Goleman D. (2000). Inteligencia emocional. Barcelona, Kairos. 25 a edición.

13. Goleman D. (1996). Emocional Intelligence: Why it can matter more than IQ. New Cork, Bantam books Psychology.

14. Hernández R. R., Fernández C. C., Baptista L.P. (2003). Metodología de la investigación. $3^{a}$ edición. Médico. Editorial McGraw-Hill. 706 pp.

15. León, C. (2001). La inteligencia emocional en estudiantes de educación superior profesional $y$ técnica de administración de empresas. V.I.G.V. Lima, Perú. Tesis de pregrado. 148 pp.

16. López P.N., Salovey P.; Straus R. Emotional Intelligence, personality and the perceived qualit of social relationships. Personality and Individual Differentes, 35 (3), 641658.2003.

17. Mayer J. Salovey P. \& Caruso D. (2000) "Emocional Intellgence as Zeitgeist, as personality, and as a mental ability". In R.
Bar - On \& D. Parker (Eds.), The Handbook of emocional intelligence (pp. 92- 117). San francisco, C.A., Jossey - Bass.

18. Mayer J. Salovey P. (1997). "What is emotional intelligence?". In P. Salovey \& D. Sluyter (Eds.), Emotional development and emotional intelligence implications for educators (pp. 3-31). New York, Básic Books.

19. Matalinares, C.M.L. et al. (2005). "Inteligencia emocional y autoconcepto en colegiales de Lima Metropolitana”. Revista de Investigación en Psicología. Volumen 8, No 01.

20. Morales A., Arcos P., Ariza E. et al. Entorno familiar y rendimiento escolar. Consejería de Educación y Ciencia de la Junta de andalucía. (Serial on line). (Citado el 15 de julio 2007). Disponible en www. Junta de Anadalucía.es/ averroes/publicaciones/investigaciones/

21. Muratta, R. (2004). Rasgos de personalidad y su relación con la inteligencia emocional en alumnos de quinto de secundaria. U.N.M.S.M. Lima,Perú. Tesis de maestría. 190 pp.

22. Olguín J. R. Inteligencia emocional. (Serial on line) (Citado el 20 de julio 2007). Disponible en http:www.uniacc.el/talon/anteriores talón_aquiles_/tal_5-1.htm.

23. Pérez, N. y Castejón, J.L. (2006). "Relaciones entre la inteligencia emocional y el cociente intelectual con el rendimiento académico en estudiantes universitarios". Revista Electrónica de Motivación y Emoción. IX. 22. Consultado el 20 de julio 2008 en http:// reme.uji.es

24. Quesada R. (1991). Guia para evaluar el aprendizaje teórico y práctico. Editorial Limusa, México.

25. Reyes, T. Y. (2002). Relación entre el rendimiento académico, la ansiedad antes de los exámenes, los riesgos de personalidad, el autoconcepto y la asertividad en estudiantes del primer año de Psicología de la UNMS. UNMS, Lima, Perú. Tesis de pregrado. 143 pp. 
26. Salovey, P \& Mayer J. (1990). "Emocional Intelligence". Imagination, Cognition and Personality. 9: 185-211.

27. Salovey P. \& Pizarro D. (2003). “The value of emocional intelligence”. In Stemberg, R., Lautrey J., Lubart T. Models of intelligence International Perspectives. Washignton D.C., American Psychological Association.

28. Sullana N. J. (1996). "La prevención del fracaso escolar, un modelo para analizar las variables que influyen en el riesgo del fracaso escolar”. Bordón. Vol. 48, No 02,151 y ss.

29. Tordike R. (1936). "Factor analisys of social and abstract intelligence". Journal of Educational Psychology. 27, 231-233.

30. Vargas, D. B. J. (2002). Factores diferenciales del rendimiento académico en educación superior. [tesis doctoral]. Universidad Anáhuac, México. 235 pp.

31. Vargas, D. B. J. (2002). Factores diferenciales del rendimiento académico en Educación Superior. Universidad Anáhuac, México. 2002. Tesis doctoral. 238 pp.
32. Velásquez, C. (2003). "La inteligencia emocional y autoestima en estudiantes de la ciudad de Lima Metropolitana con y sin participación violenta". Revista de Investigación en Psicología del Instituto de Investigaciones Psicológicos. Volumen 6, № 2. pp. 153 - 164.

33. Villacorta V. J., Villacorta V. E., Zapata V. E., Vásquez V. M., Flores R.A. (2005). El autoconcepto y el rendimiento académico en estudiantes de Derecho y Ciencias Politicas, Medicina Humana, Ciencias Agronómicas, Ciencias de la Educación de la Universidad Nacional de la Amazonía Peruana. UNAP, 2006. 